\title{
Teicoplanin potently blocks the cell entry of 2019-nCoV
}

\author{
Junsong Zhang ${ }^{1,2 \#}$, Xiancai Ma ${ }^{1 \#}$, Fei Yu ${ }^{1 \#}$, Jun Liu ${ }^{1,3}$, Fan Zou ${ }^{1,4}$, \\ Ting $\operatorname{Pan}^{1,3^{*}}$, and Hui Zhang ${ }^{1 *}$
}

1. Institute of Human Virology, Key Laboratory of Tropical Disease Control of Ministry of Education, Guangdong Engineering Research Center for Antimicrobial Agent and Immunotechnology, Zhongshan School of Medicine, Sun Yat-sen University, Guangzhou, Guangdong, China

2. Guangdong General Hospital and Guangdong Academy of Medical Sc iences, Guangzhou, Guangdong, China

3. Center for Infection \& Immunity Study, School of Medicine, Sun Yatsen University, Shenzhen, Guangdong, China

4. Guangzhou Institute of Pediatrics, Guangzhou Women and Children Medical Center, Guangzhou, Guangdong, China

\# These authors contributed equally to this work.

* To whom correspondence should be addressed:

E-mail: pant8@mail.sysu.edu.cn; zhangh92@mail.sysu.edu.cn 


\begin{abstract}
Since December 2019, the outbreak of a new coronavirus, named 2019$\mathrm{nCoV}$, has greatly threatened the public health in China and raised great concerns worldwide. No specific treatment for this infection is currently available. We previously reported that teicoplanin, a glycopeptide antibiotic which has routinely been used in the clinic to treat bacterial infection with low toxicity, significantly inhibits the invasion of cells by Ebola virus, SARS-CoV and MERS-CoV, via specifically inhibiting the activity of cathepsin L. Here, we tested the efficacy of teicoplanin against 2019-nCoV virus infection and found that teicoplanin potently prevents the entrance of 2019-nCoV-Spike-pseudoviruses into the cytoplasm, with an $\mathrm{IC}_{50}$ of $1.66 \mu \mathrm{M}$. Although the inhibitory effect upon the replication of wildtype viruses ex vivo and in vivo remains to be determined, our preliminary result indicates that the potential antiviral activity of teicoplanin could be applied for the treatment of 2019-nCoV virus infection.
\end{abstract}

Keywords: Teicoplanin, 2019-nCoV, Spike, Pseudovirus, Cathepsin L 


\section{Introduction}

The coronaviruses are enveloped, positive sense single-stranded RNA viruses ${ }^{1,2}$. The well-known examples, which have emerged as important human pathogens, include severe acute respiratory syndrome CoV (SARS$\mathrm{CoV}$ ) in China in 2003 and middle east respiratory syndrome CoV (MERS$\mathrm{CoV}$ ) in the Arabian Peninsula since $2012^{3-9}$. Recently a novel coronavirus outbreaks in December 2019 as the pathogenic agent that causes series of pneumonia cases in Wuhan of China has quickly raised intense attention not only in China but also internationally ${ }^{10-14}$. This novel coronavirus, named as 2019 novel coronavirus $(2019-\mathrm{nCoV})$, belongs to the betacoronavirus according to the sequence released ${ }^{13,15}$. Evolutionary analyses have shown that the 2019-nCoV shares $79 \%$ homology with SARS-CoV and 50\% with MERS-CoV ${ }^{15-17}$. Given the high infectious rate and the lack of effective treatment for $2019-\mathrm{nCoV}$, it is quite urgent to develop an efficient antiviral drug for 2019-nCoV.

The spike glycoprotein (S protein) is the leading mediator of viral entry, and the important determinant of host range of coronaviruses ${ }^{18}$. The infection of SARS virus is initiated by the attachment of S protein to the receptor ACE2 ${ }^{19}$, followed by cleavage with host cell protease TMPRSS2 20-22. The viruses are then transported through the early and late endosomes, subsequently endo/lysosomes, during which host protein extracellular proteases including cathepsin $\mathrm{L}$ mediates the further cleavage of S protein 
in endocytic vesicles ${ }^{23-26}$. The activated $\mathrm{S}$ protein will then activate the fusion between viral and cell membranes and release the genome of SARSCoV into cytoplasm.

In 2016, our team had found that teicoplanin, a routinely-used clinical glycopeptide antibiotics, significantly inhibited the cellular entry of Ebola virus, SARS-CoV, and MERS-CoV ${ }^{27}$. Further mechanistic studies showed that teicoplanin blocked virus entry by specifically inhibiting the activity of cathepsin L, opening a novel avenue for the development of glycopeptides as potential inhibitors of cathepsin L-dependent viruses. In this study, we have compared the cleavage site of cathepsin L in 2019$\mathrm{nCoV}$ with that in SARS-CoV and found it is well conserved. Furthermore, we identified that teicoplanin also potently inhibited the entry of 2019nCoV pseudovirus, which provide a possible strategy to the prophylaxis and treatment for2019-nCoV infection.

\section{Materials and methods}

\section{Cell culture and recombinant viruses.}

The plasmid containing spike (S) gene of 2019-nCoV was purchased from Generay Biotech company (Shanghai, China) and inserted into pcDNA3.1 vector. HIV-1/2019-nCoV-S/ pseudoviruses were produced by the co-transfection of pHIV-luciferase, psPAX2, and plasmids expressing different envelope or S proteins into HEK293T cells as 
previously described ${ }^{27}$. Briefly, the pseudoviruses including HIVluc/2019-nCoV-S, HIV-luc/SARS-CoV-S and HIV-luc/VSV-G were harvested from supernatants after 48 hours post transfection and filtered through a $0.45-\mu \mathrm{m}$ pore-size filter and stored at $-80^{\circ} \mathrm{C}$. HEK293T, A549 and Huh7 cell lines were maintained in Dulbecco's modified Eagle's medium (Gibco) with 10\% fetal calf serum (Gibco), 100 units/ml penicillin, and $100 \mu \mathrm{g} / \mathrm{ml}$ streptomycin (Gibco) at $37^{\circ} \mathrm{C}$ and $5 \% \mathrm{CO} 2$.

\section{IC50 determination.}

$\mathrm{IC}_{50}$ determination was carried out by luciferase assay from the infected cells in the presence of various concentrations (2-fold dilutions) of the teicoplanin. The $\mathrm{IC}_{50}$ curve was determined by using software from GraphPad (San Diego).

\section{siRNA transfection, RNA isolation, and RT-PCR.}

Sequences of siRNA against cathepsin Lor TMPRSS2 were predesigned by Ribobio Company (Guangzhou, China). A549 cells were seeded in $10^{5}$ cells per $\mathrm{ml}$ in each well of 12-well-microtiter plates and transiently transfected with siRNA (10-20 pmol/well) using lipofectinRNAimax reagent according to the manufacturer's instructions in serum-free medium with suitable scrambled siRNA control. Twenty-four hours later, total RNAs from the transfected cells were extracted for knocking out efficiency detection by qRT-PCR.

\section{Sequence data collection and alignment}


The genome sequences of SARS-CoV and 2019-nCoV were collected from the GenBank database (https://www.ncbi.nlm.nih.gov/nuccore/). The sequences of SARS-CoV circulating in 2003 contain 6 strains (accession number: AY278488, AY545918, AY545917, AY394977, AY394978, AY394979). The sequences of 2019-nCoV include 9 from Wuhan (MN908947, NC_045512, MN988668, MN988669, MN996527, MN996528, MN996529, MN996530, MN996531), 2 from Shenzhen (MN975262, MN938384) and 5 from USA (MN985325, MN988713, MN994467, MN994468, MN997409). The S gene sequences were obtained from the genome of SARS-CoV and 2019-nCoV according the annotation in the GenBank database. The sequence datasets were aligned using the ClustalW program implemented in MEGA X software ${ }^{28}$. Consensus sequences were created using the BioEdit software (http://www.mbio.ncsu.edu/bioedit/bioedit.html) based on the multiple alignment of SARS-CoV and 2019-nCoV, respectively. The amino acid sequence logos generated by using WebLogo ${ }^{29}$.

\section{Statistics}

Statistical analysis was performed with GraphPad Prism 7. For data with a normal distribution, we used a Student's t test. For multiple comparisons a one-way or two-way ANOVA (for parametric data) followed by Bonferroni's correction (only two groups were compared) were used. $\mathrm{P}$ values $<0.05$ were considered statistically significant. 


\section{Results}

\section{Cathepsin $L$ is required for the cell entry of 2019-nCoV.}

The proteolytic processing of the SARS-CoV S protein is essential for the virus entry and fusion. Many cleavage sites of the relevant exogenous protease including cathepsin $\mathrm{L}$ and TMPRSS2 involved in the proteolytic processing of the SARS-CoV S protein have been experimentally validated $^{20,30,31}$. Because cathepsin $\mathrm{L}$ has been identified as a target of teicoplanin, it is important to identify whether the cleavage site of cathepsin L exists on the 2019-nCOV S protein. After alignment, we found that the cleavage site of cathepsin $\mathrm{L}$ is well conserved between the SARS-CoV and 2019-nCoV S protein (Figure 1A), suggesting that cathepsin L could participant in the 2019-nCoV entry and fusion ${ }^{26,31,32}$. Moreover, the cleavage site of cathepsin $\mathrm{L}$ was consistent among the epidemic strains of 2019-nCoV retrieved from the GenBank database, including 9 from Wuhan, 2 from Shenzhen, and 5 from USA (Figure 1B). As such, we hypothesized that cathepsin L could be an important target for the entry of 2019-nCoV. Accordingly, we infected the cells with SARS-CoV-, or 2019- nCoV -S- pseudotyped HIV-1 viruses after siRNA-mediated knockdown of the expression of cathepsin L and TMPRSS2 and found that both cathepsin L and TMPRSS2 depletion impaired the cell entry of pseudoviruses (Figure 1C), indicating that both 
cathepsin L and TMPRSS2 are required for 2019- nCoV cell entry.

\section{Teicoplanin specifically inhibits the entry of 2019-nCoV.}

Based on our previous reported, we generated the HIV-luc/2019-nCoV-S pseudoviruses (Figure $2 \mathrm{~A})^{27}$. The resulting viruses were then used to infect A549 cells in the presence of teicoplanin. To exclude the possibility that the drug inhibited the early events of HIV-1 life cycle, HIV-luc/VSV-G pseudoviruses bearing vesicular stomatitis virus (VSV) glycoproteins were set up for a negative control group while pseudoviruses bearing the SARSCoV-S were used a positive $\operatorname{control}^{27}$. Here, we identified that teicoplanin acted specifically as a 2019-nCoV entry inhibitor in a dose-dependent manner (Figure 2B). It demonstrated an $\mathrm{IC}_{50}$ of $1.66 \mathrm{uM}$ for its inhibitory effect on HIV-luc/2019-nCoV-S pseudoviruses (Figure. 2C).

\section{Teicoplanin and dalbavancin repress the entry of 2019-nCoV in different cell types.}

Considering that ACE2 is a major receptor of 2019-nCoV, we have further analyzed the expression of ACE2 in different cell types from the GEO Profiles database and it was important to examine whether teicoplanin could repress the entry of 2019-nCoV viruses into different types of cells. The data showed that teicoplanin also effectively repressed virus entrance into HEK293T cells (Figure. 3A), and Huh7 cells (Figure 
3B). As we have previously demonstrated that several glycopeptide antibiotics, including teicoplanin and dalbavancin, exhibited specific inhibitory effects on cathepsin L, we therefore hypothesized that teicoplanin and its homologs could also inhibit the entry of 2019-nCoV. The data illustrated that the dalbavancin also repressed the entry of 2019$\mathrm{nCoV}$ in a dose-dependent manner (Figure 3C). However, vancomycin, another routinely-used antibiotics, did not inhibit the 2019-nCoV entry (Figure. 3D). There results were consistent with the previous inhibitory effect of several glycopeptide antibiotics on SARS-CoV and MERS$\mathrm{CoV}^{27}$

\section{Discussion}

Host cell entry is the first step of viral life cycle and is an ideal drug target for viral infection. In this study, we identified that teicoplanin could inhibit the entry of HIV-1-2019-nCoV-S pseudoviruses with the $\mathrm{IC}_{50}$ value of $1.66 \mathrm{uM}$. During the invasion phase, 2019-nCoV first binds to the receptor ACE2 on the surface of host cells. The interaction between RBD domain of $\mathrm{S}$ protein and ACE2 triggers conformational changes within $\mathrm{S}$ protein, which render the $\mathrm{S}$ protein susceptible to activation by host cell protease TMPRSS2 $20,30,33$. Subsequently, the 2019-nCoV virus enters the early endosome of the cell through endocytosis or macropinocytosis. During the early endosome maturation process, the 
endosome gradually acidifies, which has an important impact on the entry of virus into cells. The inhibitory effect of chloroquine on 2019-nCoV support this hypothesis ${ }^{17,34}$. The cysteine proteases cathepsin $\mathrm{L}$ in the endosome can further cleave the $\mathrm{S}$ protein, and activate the membrane fusion. It has been proposed that sequential cleavages of SARS-CoV S protein by TMPRSS2 and cathepsin L are necessary for fully exposure of fusion peptide at $\mathrm{S} 2$ region to the late endosome/lysosome membrane ${ }^{35}$. Our work indicates that both TMPRSS2 and cathepsin L are required for 2019-nCoV entrance, and teicoplanin potently prevents the 2019-nCoV S protein activation by directly inhibiting the enzymatic activity of cathepsin L (Figure 4) ${ }^{27}$.

Teicoplanin is a glycopeptide antibiotic, which is mainly used for serious infections caused by Gram-positive bacteria such as staphylococcus aureus and streptococcus ${ }^{36-38}$. As a routinely-used clinical antibiotics, teicoplanin is well known for its much low toxic and side effects, long half-life in blood plasma, convenient administration, and high safety when used in combination with other antibiotics. The recommended plasma concentration of teicoplanin for clinical use to inhibit Gram-positive bacteria is $15 \mathrm{mg} / \mathrm{L}$, or $8.78 \mu \mathrm{M}$, and the commonly used dose is $400 \mathrm{mg}$ / day. Here we found the $\mathrm{IC}_{50}$ inhibition of 2019-nCOV was only $1.66 \mathrm{uM}$, which is much lower than the routine clinical drug concentration. Therefore, the routinely-used dose (400 mg / 
day) could be considered for patients with 2019-nCOV infection. If the effect is not significant, dose could be optimized because of its low toxicity. The doses such as $800 \mathrm{mg} /$ day or $1200 \mathrm{mg} /$ day could be considered to improve the drug efficiency. Given that the principle of antiviral therapy is to prevent virus infection and amplification at a stage as early as possible, it is reasonable to recommend the use of teicoplanin for 2019-nCoV in the early stage. Alternatively, it could substitute vancomycin or other antibiotics to treat the co-infection with Grampositive bacteria at a proper time. As such, teicoplanin could function as a dual inhibitor for both the 2019-nCOV infection and co-infection with Gram-positive bacteria.

\section{Disclosure statement}

No potential conflict of interest was reported by the authors.

\section{Funding}

This study was supported by National Key Research and Development Program of China (2020YFC0841400), the National Special Research Program of China for Important Infectious Diseases (2018ZX10302103, 2017ZX10202102-003), the Important Key Program of Natural Science Foundation of China (81730060), and the Joint-Innovation Program in Healthcare for Special Scientific Research Projects of Guangzhou 
(201803040002) to H.Z. This work was also supported by the Pearl River

S\&T Nova Program of Guangzhou (201806010118) and the National

Natural Science Foundation of China (81971918) to T.P. 


\section{Figure legends}

\section{Figure 1. Cathepsin $L$ is required for the entry of 2019-nCoV.}

$\mathrm{A}$, The sequence alignment based on the consensus $\mathrm{S}$ protein sequences of SARS-CoV and 2019-nCoV. Alignment was performed by using ClustalW method. The amino acid sequence logos generated by using WebLogo was the graphical representation of the multiple alignment of the sequences of SARS-CoV and 2019-nCoV. The overall height of the stack indicated the sequence conservation at that position, while the height of symbols within the stack indicated the relative frequency (Y-axis) of each amino acid at that position (X-axis).

$\mathrm{B}$, The multiple alignment created based on the region containg in the cleavage site of cathepsin L (SIIAYTMSLGA) on the S protein of 2019nCoV, including 9 from Wuhan, 2 from Shenzhen and 5 from USA. The accession number of each sequence was showed in the strain name. The identity/similarity shading with the color was referred to the chemistry of each amino acid at that position.

C, HEK293T cells were transfected with $200 \mathrm{nM}$ siRNAs per well. After $24 \mathrm{~h}$, the cells were infected with HIV-luc/2019-nCoV pseudoviruses. After washing and incubation with fresh medium for $48 \mathrm{~h}$, the intracellular luciferase activity was measured. Ordinary one-way ANOVA test was used for this analysis. The results are shown as the mean and SEM. $* \mathrm{P}<0.05$. 


\section{Figure 2. Teicoplanin specifically inhibits the entry of 2019-nCoV.}

A, Schematic representation of pseudovirus entry assay.

B, Teicoplanin inhibit the entry of 2019-nCoV and SARS-nCoV. Chemical structure of teicoplanin (Left). A549 cells were seeded in a 96-well plate, and $24 \mathrm{~h}$ later, the cells were infected with HIV-luc/2019-nCoV, HIVluc/SARS-nCoV or HIV-luc/VSVG pseudoviruses. After washing and incubation with fresh medium for $48 \mathrm{~h}$, the intracellular luciferase activity was measured. Ordinary one-way ANOVA test was used for this analysis. The results are shown as the mean and SEM. ${ }^{*} \mathrm{P}<0.05$.

C, A549 cells were infected with HIV-luc/2019-nCoV pseudoviruses and then incubated with teicoplanin at various concentrations. The intracellular luciferase activity was measured at $48 \mathrm{~h}$ post-infection. The $\mathrm{IC}_{50}$ was calculated using GraphPad Prism software.

Figure 3. Teicoplanin and dalbavancin but not vancomycin repress the entry of 2019-nCoV into different cell types.

A, HEK293T cells were infected with HIV-luc/2019-nCoV pseudoviruses and then incubated with teicoplanin at various concentrations. The intracellular luciferase activity was measured at $48 \mathrm{~h}$ post-infection. The $\mathrm{IC}_{50}$ was calculated using GraphPad Prism software.

B, Huh7 cells were infected with HIV-luc/2019-nCoV pseudoviruses and then incubated with teicoplanin at various concentrations. The intracellular 
luciferase activity was measured at $48 \mathrm{~h}$ post-infection. The $\mathrm{IC}_{50}$ was calculated using GraphPad Prism software.

C and D, A549 cells were incubated with vancomycin (C) or dalbavancin (D) at various concentrations and infected with HIV-luc/2019-nCoV pseudoviruses. The intracellular luciferase activity was measured at $48 \mathrm{~h}$ post-infection. Ordinary one-way ANOVA test was used for this analysis. Left: Chemical structure of vancomycin (A) or dalbavancin (B).

The results are shown as the mean and SEM. $* \mathrm{P}<0.05$.

Figure 4. The schematic of teicoplanin blocking the entry of 2019nCoV.

Schema graph of the 2019-nCoV for entry into target cells. After binding of virus to the cellular receptor ACE2, the proteolytic process was initialed by TMPRSS2 on the cellular membrane. The virions will be up-taken into endosomes, where the $\mathrm{S}$ protein is further activated by cleavage with cysteine protease cathepsin L. The cleavage of S protein by cathepsin L can be significantly blocked by teicoplanin. 


\section{Reference}

1 Cui, J., Li, F. \& Shi, Z. L. Origin and evolution of pathogenic coronaviruses. Nat Rev Microbio/17, 181-192, doi:10.1038/s41579-018-0118-9 (2019).

2 Chen, Y., Liu, Q. \& Guo, D. Emerging coronaviruses: genome structure, replication, and pathogenesis. J Med Virol, doi:10.1002/jmv.25681 (2020).

3 Chan, J. F., To, K. K., Tse, H., Jin, D. Y. \& Yuen, K. Y. Interspecies transmission and emergence of novel viruses: lessons from bats and birds. Trends Microbio/ 21, 544-555, doi:10.1016/j.tim.2013.05.005 (2013).

4 Lu, G., Wang, Q. \& Gao, G. F. Bat-to-human: spike features determining 'host jump' of coronaviruses SARS-CoV, MERS-CoV, and beyond. Trends Microbiol 23, 468-478, doi:10.1016/j.tim.2015.06.003 (2015).

5 de Wit, E., van Doremalen, N., Falzarano, D. \& Munster, V. J. SARS and MERS: recent insights into emerging coronaviruses. Nature Reviews Microbiology 14, 523-534, doi:10.1038/nrmicro.2016.81 (2016).

6 Lau, S. K. et al. Severe acute respiratory syndrome coronavirus-like virus in Chinese horseshoe bats. Proc Natl Acad Sci U S A 102, 14040-14045, doi:10.1073/pnas.0506735102 (2005).

7 Zaki, A. M., van Boheemen, S., Bestebroer, T. M., Osterhaus, A. D. \& Fouchier, R. A. Isolation of a novel coronavirus from a man with pneumonia in Saudi Arabia. N Eng/ J Med 367, 1814-1820, doi:10.1056/NEJMoa1211721 (2012).

8 Peiris, J. S., Yuen, K. Y., Osterhaus, A. D. \& Stohr, K. The severe acute respiratory syndrome. N Eng/ J Med 349, 2431-2441, doi:10.1056/NEJMra032498 (2003).

9 Peiris, J. S. et al. Coronavirus as a possible cause of severe acute respiratory syndrome. Lancet 361, 1319-1325, doi:10.1016/s0140-6736(03)13077-2 (2003).

Horton, R. Offline: 2019-nCoV outbreak-early lessons. Lancet 395, 322, doi:10.1016/S0140-6736(20)30212-9 (2020).

11 Lu, R. et al. Genomic characterisation and epidemiology of 2019 novel coronavirus: implications for virus origins and receptor binding. Lancet, doi:10.1016/S01406736(20)30251-8 (2020).

12 Rothe, C. et al. Transmission of 2019-nCoV Infection from an Asymptomatic Contact in Germany. N Eng/ J Med, doi:10.1056/NEJMc2001468 (2020).

13 Zhu, N. et al. A Novel Coronavirus from Patients with Pneumonia in China, 2019. N Eng/ J Med, doi:10.1056/NEJMoa2001017 (2020).

14 Huang, C. et al. Clinical features of patients infected with 2019 novel coronavirus in Wuhan, China. Lancet, doi:10.1016/S0140-6736(20)30183-5 (2020).

15 Wu, F. et al. A new coronavirus associated with human respiratory disease in China. Nature, 10.1038/s41586-41020-42008-41583, doi:10.1038/s41586-020-2008-3 (2020).

16 Chan, J. F. et al. Genomic characterization of the 2019 novel human-pathogenic coronavirus isolated from a patient with atypical pneumonia after visiting Wuhan. Emerg Microbes Infect 9, 221-236, doi:10.1080/22221751.2020.1719902 (2020).

17 Zhou, P. et al. A pneumonia outbreak associated with a new coronavirus of probable bat origin. Nature, 10.1038/s41586-41020-42012-41587, doi:10.1038/s41586-020-2012-7 (2020). 
Gallagher, T. M. \& Buchmeier, M. J. Coronavirus spike proteins in viral entry and pathogenesis. Virology 279, 371-374, doi:10.1006/viro.2000.0757 (2001).

$19 \mathrm{Li}, \mathrm{W}$. et al. Angiotensin-converting enzyme 2 is a functional receptor for the SARS coronavirus. Nature 426, 450-454, doi:10.1038/nature02145 (2003).

20 Glowacka, I. et al. Evidence that TMPRSS2 Activates the Severe Acute Respiratory Syndrome Coronavirus Spike Protein for Membrane Fusion and Reduces Viral Control by the Humoral Immune Response. Journal Of Virology 85, 4122-4134, doi:10.1128/Jvi.02232-10 (2011).

21 Shulla, A. et al. A transmembrane serine protease is linked to the severe acute respiratory syndrome coronavirus receptor and activates virus entry. J Virol 85, 873-882, doi:10.1128/JVI.02062-10 (2011).

Reinke, L. M. et al. Different residues in the SARS-CoV spike protein determine cleavage and activation by the host cell protease TMPRSS2. PLOS One 12, e0179177, doi:10.1371/journal.pone.0179177 (2017).

Matsuyama, S., Ujike, M., Morikawa, S., Tashiro, M. \& Taguchi, F. Protease-mediated enhancement of severe acute respiratory syndrome coronavirus infection. 102, 1254312547, doi:10.1073/pnas.0503203102J Proceedings of the National Academy of Sciences of the United States of America (2005).

24 Belouzard, S., Chu, V. C. \& Whittaker, G. R. Activation of the SARS coronavirus spike protein via sequential proteolytic cleavage at two distinct sites. 106, 5871-5876, doi:10.1073/pnas.0809524106 \%] Proceedings of the National Academy of Sciences (2009). Huang, I. C. et al. SARS coronavirus, but not human coronavirus NL63, utilizes cathepsin $\mathrm{L}$ to infect ACE2-expressing cells. J Biol Chem 281, 3198-3203, doi:10.1074/jbc.M508381200 (2006).

Simmons, G. et al. Inhibitors of cathepsin L prevent severe acute respiratory syndrome coronavirus entry. Proc Natl Acad Sci U S A 102, 11876-11881, doi:10.1073/pnas.0505577102 (2005).

Zhou, N. et al. Glycopeptide Antibiotics Potently Inhibit Cathepsin L in the Late Endosome/Lysosome and Block the Entry of Ebola Virus, Middle East Respiratory Syndrome Coronavirus (MERS-CoV), and Severe Acute Respiratory Syndrome Coronavirus (SARS-CoV). Journal Of Biological Chemistry 291, 9218-9232, doi:10.1074/jbc.M116.716100 (2016).

28 Kumar, S., Stecher, G., Li, M., Knyaz, C. \& Tamura, K. MEGA X: Molecular Evolutionary Genetics Analysis across Computing Platforms. Mol Biol Evol 35, 1547-1549, doi:10.1093/molbev/msy096 (2018).

29 Crooks, G. E., Hon, G., Chandonia, J. M. \& Brenner, S. E. WebLogo: a sequence logo generator. Genome Res 14, 1188-1190, doi:10.1101/gr.849004 (2004).

30 Simmons, G., Zmora, P., Gierer, S., Heurich, A. \& Pöhlmann, S. Proteolytic activation of the SARS-coronavirus spike protein: Cutting enzymes at the cutting edge of antiviral research. Antiviral Research 100, 605-614, doi:https://doi.org/10.1016/j.antiviral.2013.09.028 (2013).

31 Bosch, B. J., Bartelink, W. \& Rottier, P. J. Cathepsin L functionally cleaves the severe acute respiratory syndrome coronavirus class I fusion protein upstream of rather than adjacent to the fusion peptide. J Viro/ 82, 8887-8890, doi:10.1128/JVI.00415-08 (2008). 
32 Belouzard, S., Millet, J. K., Licitra, B. N. \& Whittaker, G. R. Mechanisms of coronavirus cell entry mediated by the viral spike protein. Viruses 4, 1011-1033, doi:10.3390/v4061011 (2012).

33 Hoffmann, M. et al. The novel coronavirus 2019 (2019-nCoV) uses the SARS-coronavirus receptor ACE2 and the cellular protease TMPRSS2 for entry into target cells. 2020.2001.2031.929042, doi:10.1101/2020.01.31.929042\%] bioRxiv (2020).

34 Wang, M. et al. Remdesivir and chloroquine effectively inhibit the recently emerged novel coronavirus (2019-nCoV) in vitro. Cell Research, doi:10.1038/s41422-020-0282-0 (2020).

35 Belouzard, S., Chu, V. C. \& Whittaker, G. R. Activation of the SARS coronavirus spike protein via sequential proteolytic cleavage at two distinct sites. Proceedings of the National Academy of Sciences of the United States of America 106, 5871-5876, doi:10.1073/pnas.0809524106 (2009).

36 Parenti, F., Beretta, G., Berti, M. \& Arioli, V. Teichomycins, new antibiotics from Actinoplanes teichomyceticus Nov. Sp. I. Description of the producer strain, fermentation studies and biological properties. J Antibiot (Tokyo) 31, 276-283, doi:10.7164/antibiotics.31.276 (1978).

37 Borghi, A. et al. Teichomycins, new antibiotics from Actinoplanes teichomyceticus nov. sp. IV. Separation and characterization of the components of teichomycin (teicoplanin). J Antibiot (Tokyo) 37, 615-620, doi:10.7164/antibiotics.37.615 (1984).

Shea, K. W. \& Cunha, B. A. Teicoplanin. Med Clin North Am 79, 833-844, doi:10.1016/s0025-7125(16)30042-6 (1995). 
bioRxiv preprint doi: https://doi.org/10.1101/2020.02.05.935387; this version posted February 13, 2020. The copyright holder for this preprint (which was not certified by peer review) is the author/funder. All rights reserved. No reuse allowed without permission.

Fig.1

A

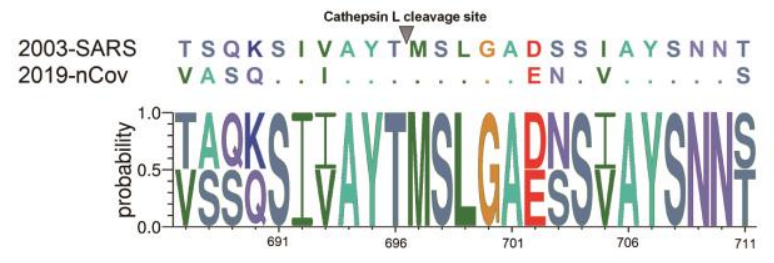

C

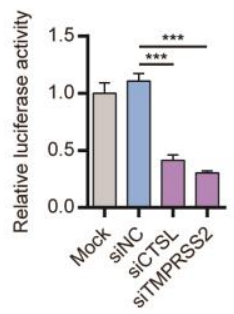

B

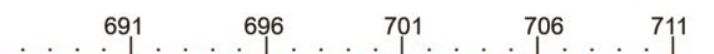

Wuhan|MN908947

Wuhan|NC_045512

Wuhan|MN988668

Wuhan|MN988669

Wuhan|MN996527

Wuhan|MN996528

Wuhan|MN996529

Wuhan|MN996530

Wuhan|MN996531

Shenzhen|MN938384

Shenzhen|MN975262

USAIMN985325

USA|MN988713

USAIMN994467

USA|MN994468

USA|MN997409

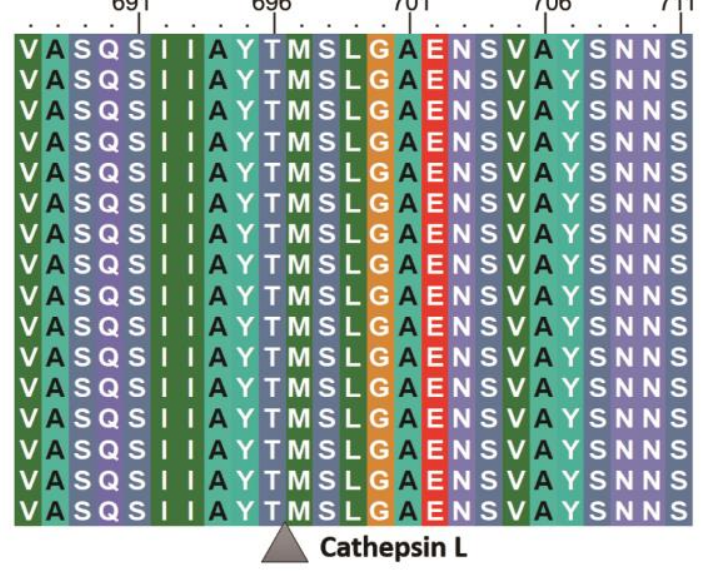


bioRxiv preprint doi: https://doi.org/10.1101/2020.02.05.935387; this version posted February 13, 2020. The copyright holder for this preprint (which was not certified by peer review) is the author/funder. All rights reserved. No reuse allowed without permission.

Fig.2

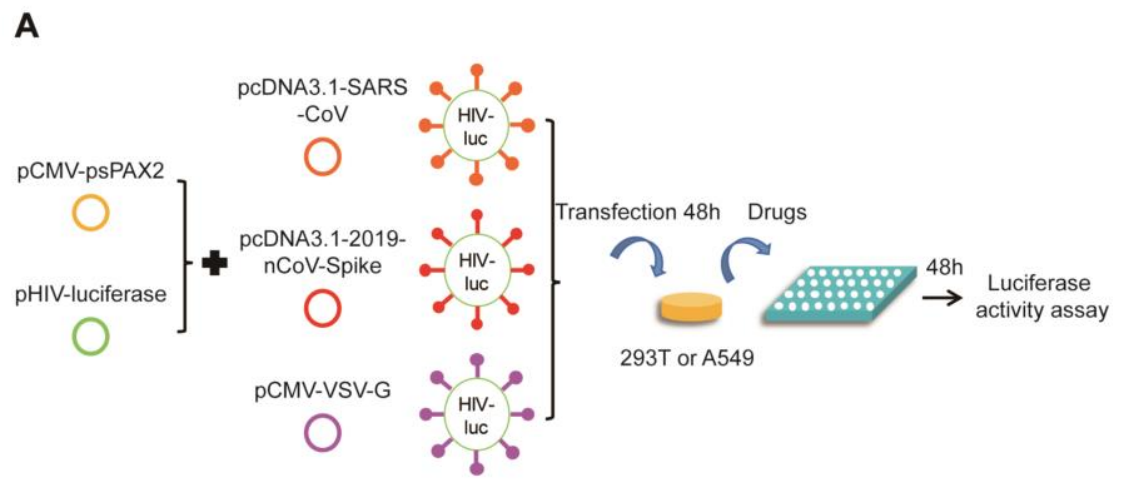

B
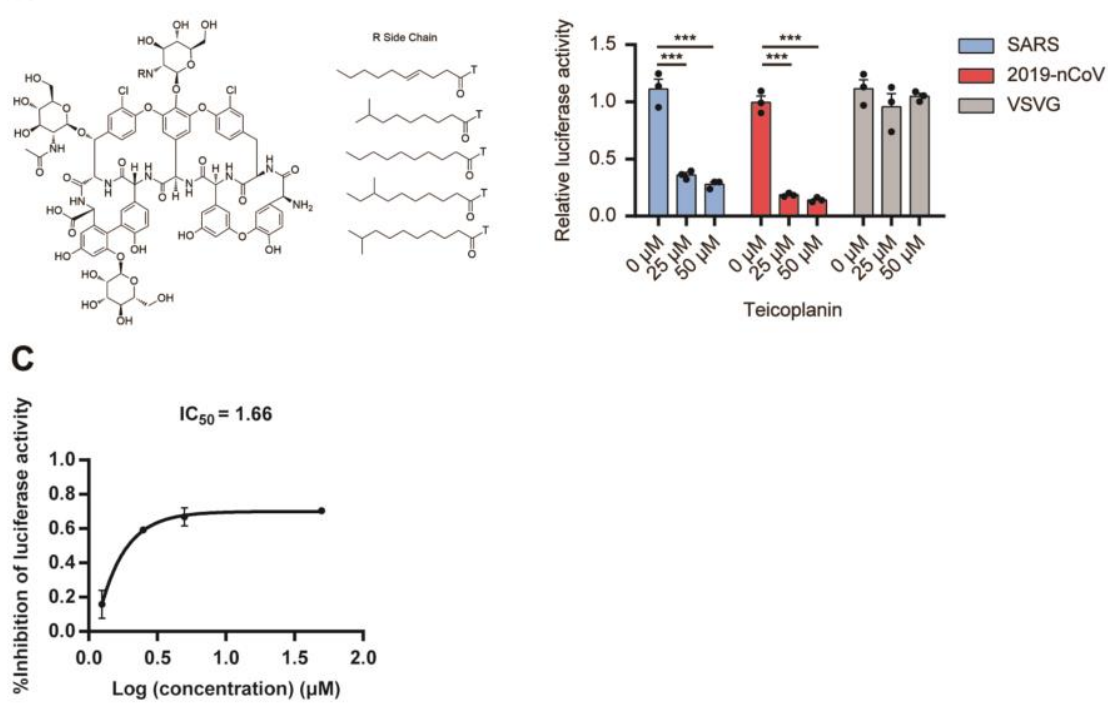
Fig.3

A

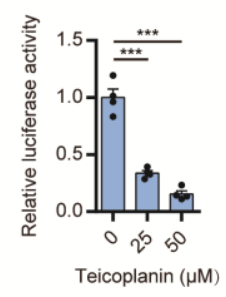

B

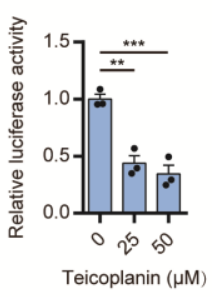

C
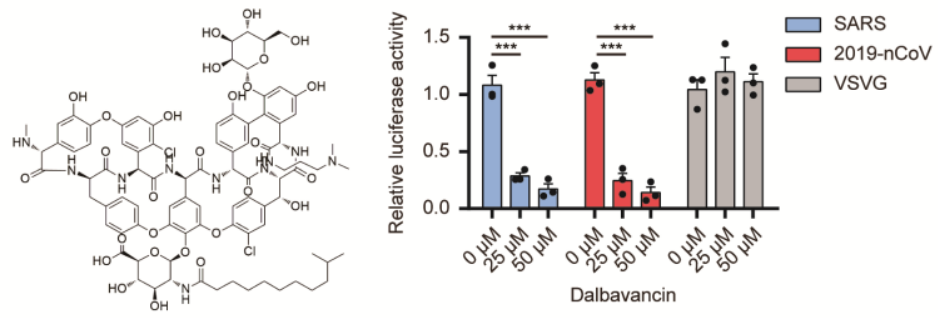

D
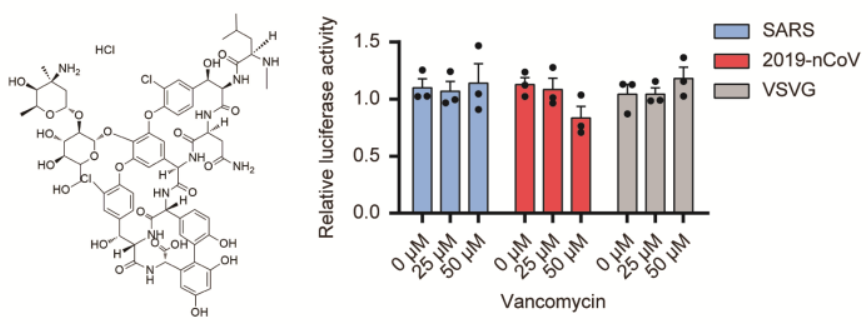
Fig.4

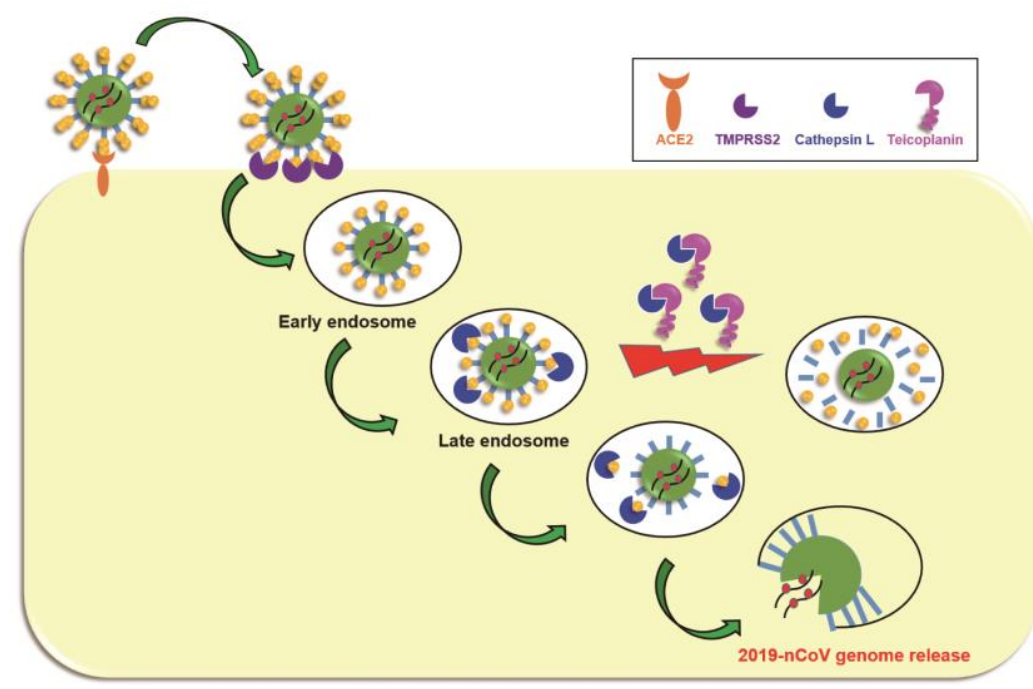

